\title{
Indicador de Medición en Psicología online en América Latina en tiempos de pandemia
}

\section{Measurement indicator to psychology online in Latin American in pandemic times}

Carlos Andrés Trejos-Gil. Universidad Católica Luis Amigó. Colombia.

carlos.trejosgi@amigo.edu.co

$[\mathrm{CV}]$ O $\mathrm{G} \mathrm{R}^{\mathrm{O}} \mathrm{C}$

Huber Yesid Castro Escobar. Universidad Católica Luis Amigó. Colombia.

huber.castroes@amigo.edu.co

$[\mathrm{CV}](\mathrm{C})$

Omar Augusto Amador Sánchez. Universidad Católica Luis Amigó. Colombia.

omar.amadorsa@amigo.edu.co

$[\mathrm{CV}]$ (1)

Cómo citar este artículo / Referencia normalizada

Trejos-Gil, C. A., Castro-Escobar, H. y Amador S. (2020). Indicador de Medición en Psicología online en América Latina en tiempos de pandemia. Revista Latina de Comunicación Social, 78, 457-476. https://www.doi.org/10.4185/RLCS-2020-1485

\section{RESUMEN}

Introducción: En ámbitos de tecnología, virtualización y digitalización se brindan ciberespacios de oferta y demanda de servicios vía online; uno de ellos en tiempos de pandemia, es la posibilidad de adquirir servicios de psicología vía online, asumiendo a priori que estos sitios web son de confianza para los usuarios y de buena calidad. Este trabajo propone ciertos indicadores para evaluar los sitios web que prestan el servicio de psicología online en América latina. Metodología: Bajo un diseño metodológico experimental se analizó 144 sitios web de psicología online evaluando variables objetivas para someterlas a un análisis factorial con el propósito de la construcción de indicadores. Resultados: Según los indicadores propuestos se identifica a Brasil $(\bar{X}=2.00)$ como el país que mejor índices arrojó, seguido de Argentina $(\bar{X}=2.5)$, Chile $(\bar{X}=2.67)$ y Colombia $(\bar{X}=3.5)$; Integra Médica (Chile) (IMPOL=0.4629) es la URL de psicología online que mejor calificación obtuvo en América Latina. Discusión: A pesar de las condiciones pandémicas que atraviesa el mundo actualmente, y la latente necesidad de las personas para acceder a terapias psicológicas online, los sitios web carecen de varios aspectos de calidad web como seguridad, accesibilidad y posicionamiento web. Conclusiones: El Indicador de Medición de Psicología Online (IMPOL) es una herramienta que permite evaluar a los sitios web de esta categoría y detectar fortalezas y debilidades de cada sitio que brinda psicología online en tiempos de pandemia.

PALABRAS CLAVE: psicología; epidemia; análisis factorial; América latina; comercio electrónico; seguridad; accesibilidad a la información. 


\begin{abstract}
Introduction: In the areas of technology and virtualization from provide online services in pandemic times there is a possibility psychology online acquiring services thinking that web sites are confidence for users and good quality. This paper proposes a number of indicators to websites evaluation that provide online services psychology in Latin American. Methodology: Whit experimental methodology were tracked 144 websites online services psychology assessing objectives variables with the purpose of factor analysis to the indicators building. Results: According the proposed indicators Brazil $(\bar{X}=2.00)$ is identified that scored best indicator, followed by Argentina $(\bar{X}=2.5)$, Chile $(\bar{X}=2.67)$ y Colombia $(\bar{X}=3.5)$; Integra Medical (Chile) (IMPOL=0.4629) get the better calification in Latin America, Discussion: Despite the pandemics conditions of the world actually, latent need the persons to access psychology online therapy, the web site aspects lacking to web quality with safety, accessibility and web positioning. Conclusions: Measurement indicator to psychology online (MIPOL) is a tool that permit to evaluate websites this category and detected strengths and weaknesses each web sites.
\end{abstract}

KEYWORDS: psychology; epidemic; factor analysis, Latin America, electronic commerce; security; information accessibility.

\title{
CONTENIDO
}

1. Introducción. 2. Metodología. 2.1. Participantes - Muestra poblacional. 2.2. Instrumento. 2.3 Procedimiento. 2.4. Análisis estadístico 3. Resultados. 3.1. Indicador de Medición en Psicología Online. 4. Discusión. 5. Conclusiones. 6. Bibliografía.

\section{Introducción}

En el marco de la ciencia que estudia todos aquellos procesos mentales, capacidades humanas, percepciones y los comportamientos del ser desde una disciplina analítica como lo es la psicología, que en otras palabras estudia la actividad mental básicamente desde las perspectivas, conductual, afectiva y cognitiva, se presentan diferentes fenómenos socioculturales que de una u otra forma permiten que cambien las formas tradicionales de ejercer las ciencias, en este caso, sobre las formas tradicionales del servicio psicológico en que las personas acceden a terapias personalmente, y que se enfrentan a menor tiempo para tener sus citas personales con su psicólogo de preferencia.

El avance estrepitoso de la tecnología que invade indefectiblemente cualquier área del saber, en los últimos tiempos se encuentran diferentes vertientes de las ciencias y en específico el estudio de la mente y sus procesos psíquicos; cada vez con mayor validez que se impregne por aparatos, herramientas y medios que facilitan el contacto y mejoran las dinámicas que la psicología tiene en medio de sus procedimientos terapéuticos. Actualmente se cuenta con servicios de e-Salud (BouzasLorenzo \& Mahou, 2013 y Organización Panamericana de la Salud, 2016; Sanmartín, Ávila, Vilora \& Jabba, 2016) servicios de salud mediadas por la internet, también denominada como telesalud o telemedicina por el uso de las tecnologías, videoconferencias, e-mail y Smartphone apps, para apoyar los servicios de salud a distancia (Yang, \& Zhang, 2019; Zhou et al., 2020), son estos mecanismos predilectos para afrontar un distanciamiento social para obtener sesiones psicológicas a distancia.

La psicología no está ajena a la adaptación de los nuevos ejercicios terapéuticos mediados por la web, donde "la utilización de los medios de comunicación de masas para divulgar la psicología han acarreado consecuencias ambivalentes para tal disciplina” (Arana, Meilán, \& Pérez, 2006); es por esta razón que yace la inquietud en esta investigación, sobre el análisis de la prestación del servicio 
psicológico de forma virtual, acortando las distancias, minimizando las barreras, incluso minimizando precios de venta, facilitando tareas y actividades de los usuarios (Echeburúa \& de Corral, 2010) permitiendo el acceso de manera eficaz y dinámica para ejercer la psicología a un público ávido por este servicio, teniendo en cuenta fenómenos de salud pública como la crisis actual de pandemia del COVID-19, y como las nuevas formas de interacción en las nuevas comunidades en línea (Castells, 2009), y que el uso de estas tecnologías en la sociedad moderna no causen un efecto contrario convirtiéndolas en un fin y no en un medio (Espinar, Zych, \& Rodríguez-Hidalgo, 2015).

Un estudio realizado por We Are Social no solo muestra el uso cuantitativo del uso de la internet, sino, también la gran posibilidad de generar oferta de servicios a tanto público dispuesto a pagar por ello, aunque los niveles de calidad puedan verse perjudicados, pues aunque no se cuestiona el crecimiento de la oferta de servicios psicológicos a través de la web, el uso el teléfono fijo en un principio y luego el móvil, se convirtieron en herramientas ideales para prestar servicios de ePsicología (Baena, Quesada \& Vogt, 2008); luego este ejercicio incursionó por medio de la web, teniendo en cuenta la internet como medio para compartir experiencias y comunicarse con otros (Ortega Ruíz, Del Rey, \& Sánchez, 2012). En la actualidad, hasta se ha implementado la realidad virtual y realidad aumentada como simulación de situaciones para ejercicios terapéuticos (Soto \& Gómez, 2018; Baena, Quesada \& Vogt, 2008 y Brown, 1998) en el campo de la e-psicología o tele medecina, así las videollamadas han mediado ejercicios terapéuticos y reducen el aislamiento social y la soledad durante la crisis actual, sobre todo en adultos mayores (Noone et al., 2020); del mismo modo, en tiempos de pandemia COVID-19 estudios develan la inteligencia artificial (IA) como herramientas efectivas en programas de intervención en crisis psicológicas, así mismo los servicios de salud mental online facilitan las intervenciones de emergencia y mejoran su calidad en la actual epidemia (Liu, et al., 2020).

Los profesionales de la psicología no pueden estar ajenos a los avances tecnológicos más allá de tener conocimientos en su profesión, y si quieren ofertar sus servicios a través del ciberespacio (Domínguez, 2007), estos deben tener presente aspectos periféricos a su profesión como, "organización, integridad, remuneración y flexibilidad" (Helou et al., 2020); además, debe contar con conocimientos en "Soportes tecnológicos para la gestión documental; gestión de contenido web en redes sociales; web semántica para productos de valor añadido y posibilidades de negocio con el tratamiento de la información." (Morato, Sánchez \& Fernández, 2015); y además, fortalecer sus competencias denominadas "Interés tecnológico" y "e-Psicología" como uso de las TIC para ponerse en contacto con sus pacientes (Brown, 1998; Charria, Sarsosa , \& Arenas, 2011).

Esta investigación pretende levantar un diagnóstico sobre la oferta de servicios psicológicos mediados por la internet puntualmente en latinoamérica; qué características tienen estas plataformas que ofrecen tal servicio; qué patrones de estructura web tienen y si existe una forma de medir y caracterizar estas plataformas web. Existe una variedad de metodologías para analizar web (Hernández \& Fransi, 2014; Codina, et al., 2014; Rodríguez-Martínez, Codina, \& Pedraza-Jiménez, 2010 y Freixa, Soler-Adillon, Sora \& Ribas, 2014) y que son pertinentes para este estudio en concreto. Primero que todo estos estudios se refieren al análisis de cibermedios y en otros estudios se habla de metamedios, pero para este estudio el término cibermedios es el más adecuado.

El sistema SAAC por Codina et al. (2014) ilustra mediante dos conjuntos (C. de Términos y C. de Fases, Actividades y Resultados) un sistema para estudiar medios de comunicación digitales, articulando el primer conjunto que se constituye de 12 Términos con el segundo conjunto constituído por 4 Fases, 11 Actividades y los Resultados por cada Fase; lo interesante de esta metodología es que es abierta y libre a aplicación de cualquier cibermedio con las variables que el investigador considere pertinentes. Por otro lado, está el estudio por Rodríguez-Martínez, Codina, y Pedraza-Jiménez (2010) 
sobre Cibermedios y Web 2.0 para determinar su calidad en relación a 3 indicadores (Generales, Específicos Internos y Específicos Externos) en términos de accesibilidad (Valdés, 2013; Conesa, Aguinaga, Hernández, 2011 y Voces-Merayo, 2007), visibilidad y popularidad, acceso a la información (Cristobal-Fransi \& Marimon-Viadiu, 2010; Vázquez-Casielles, Rio-Lanza \& SuárezÁlvarez, 2009) de 3 categorías y tecnologías que facilitan la comunicación social.

Sin embargo, Hernández y Fransi (2014) describen con sus 4 dimensiones como un método de evaluación de la calidad en los cibermedios; dimensiones como eficiencia (Petnji, Marimon \& Casadesús, 2012), disponibilidad del sistema, fiabilidad y privacidad (Parasuraman, Zeithaml. \& Malhotra, 2005 y Vázquez-Casielles, Rio-Lanza \& Suárez-Álvarez, 2009) e interacción, características que hacen una evaluación completa desde cada una de sus propias variables. Este estudio y los mencionados anteriormente en conjunto complementan la metodología de análisis de cibermedios en la prestación de servicios en psicología online de una manera completa, global y sistémica como se pretende.

Por otra parte, la medición de los sitios web ha estado bastante investigada sin generar aún un modelo práctico o un constructo en términos de calidad que permita evaluar generalmente a los sitios web bajo un mismo parámetro o instrumento (González, Bañegil \& Buenadicha, 2013); estudios como el de Rappa (2005) proponen que exiten diferentes tipos de sitios web estableciendo 9 categorías, corretaje, publicidad, infomediario, comerciante, fabricante, afiliación, comunidad, suscripción y utilidad. En ese sentido los sitios web que ofertan servicios de psicología vía online caben dentro del tipo comerciante bajo el criterio de Rappa (2005).

La norma ISO9126 del mismo modo comparte un modelo de calidad de software en 3 importantes áreas, interior (propiedades estáticas del código), exterior (propiedades dinámicas del código al ejecutarse) y en uso (satisfacción de necesidades al usuario). La calidad del software se expresa en 10 características (las 6 primeras de ellas comunes entre internas y externas y 4 de uso); estas características son: Funcionalidad; Fiabilidad; Usabilidad; Eficiencia; Mantenibilidad; Portabilidad; Efectividad; Productividad; Seguridad y Satisfacción. Estudios recientes demuestran el análisis web/software los cuales se refieren en términos de calidad, como e-SQ/e-SERVQUAL/e-QUAL; WebQUAL; SiteQUAL; ComQ/eTailQ; WebQUAL 4.0 y Etransqual en los que se miden mediante una escala los aspectos "Eficiencia, Cumplimiento, Disponibilidad, Privacidad, Capacidad de respuesta, Compensación, Contacto, Utilidad, Facilidad de uso, Diseño, Entretenimiento, Relación complementaria, Rapidez de procesamiento, Seguridad, Diseño del sitio web, Fiabilidad/cumplimiento, Privacidad/seguridad, Atención al cliente, Usabilidad, Calidad de información, Calidad de interacción, Funcionalidad/diseño, Disfrute, Proceso, Fiabilidad y Capacidad de respuesta" (González et al., 2013; Zeithaml, 2000, 2002; Zeithaml et al., 2002; Parasuraman et al., 2005; Loiacono et al., 2007; Yoo \& Donthu, 2001; Wolfinbarger \& Gilly, 2003 Barnes \& Vidgen, 2002; Petnji et al., 2012; Palacios \& Noci, 2008; y Bauer \& Scharl, 2006).

Y si la teoría indica diferentes estudios de análisis web, y teniendo en cuenta que no existe una fórmula exacta o modelo general que evidencie una evaluación profunda de los sitios web, se puede entonces proponer que se evalúen los sitios web que oferten servicios de psicología online en tiempos de pandemia, cuando miles y miles de personas confinadas por decretos de gobierno deben estar encerradas en sus casas por largos periodos de tiempo, cuando se ponen a prueba la tolerancia, empatía y otros valores que deben surgir en estas condiciones de cuarentenas sociales. Y es allí, donde muchas personas recurren a los servicios de psicología online; pero no se tienen estudios verídicos que confirmen la presencia de tales sitios, que ofrezcan garantías para los usuarios, que brinden confiabilidad y sobre todo privacidad de los datos personales. Y cómo no encontrar oferta psicológica en la web aunque se planteen dudas sobre la regulación de estos nuevos modelos 
virtuales terapéuticos, la verificación de calidad de la prestación del servicio teniendo en cuenta la pseudo-información, por tal razón se requieren estudios que determinen cuál es el panorama actual de cada país frente a la oferta de este servicio en psicología online en tiempos de pandemia, a razón que, aún no se registran estudios de relevancia con relación al presente objeto de estudio.

Por estas razones fundamentadas en la teoría, es necesario analizar los sitios web que brindan servicios de psicología online en latinoamérica, y proponer mediante este estudio un indicador genérico para los sitios web de esta categoría en la prestación de servicios psicológicos.

\section{Metodología}

\subsection{Participantes - Muestra poblacional}

El presente estudio se basa sobre un método cuantitativo con validación estadística aplicado en una población total $(\mathrm{N})$ de 144 cibermedios en la prestación de servicios de psicología, que para el estudio se denomina ciberpsicología. Todos los cibermedios analizados cuentan con la característica principal de sitio web de América Latina.

Tabla 1. Distribución poblacional de cibermedios en ciberpsicología.

\begin{tabular}{llllllll}
\hline \multicolumn{1}{c}{ País } & \multicolumn{1}{c}{ Cant. } & \%. & Zona G. & \multicolumn{1}{c}{ País } & Cant. & \% & Zona G. \\
\hline Argentina & 26 & $18,1 \%$ & S.A & Honduras & 4 & $2,8 \%$ & C.A \\
Bolivia & 5 & $3,5 \%$ & S.A & México & 14 & $9,7 \%$ & N.A. \\
Brasil & 22 & $15,3 \%$ & S.A & Panamá & 4 & $2,8 \%$ & C.A \\
Chile & 16 & $11,1 \%$ & S.A & Paraguay & 2 & $1,4 \%$ & S.A \\
Colombia & 28 & $19,4 \%$ & S.A & Perú & 5 & $3,5 \%$ & S.A \\
Costa Rica & 1 & $0,7 \%$ & C.A & Puerto Rico & 2 & $1,4 \%$ & N.A. \\
Ecuador & 2 & $1,4 \%$ & S.A & Rep. Dominic. & 2 & $1,4 \%$ & C.A \\
El Salvador & 2 & $1,4 \%$ & C.A & Uruguay & 3 & $2,1 \%$ & S.A \\
Guatemala & 4 & $2,8 \%$ & C.A & Venezuela & 2 & $1,4 \%$ & S.A \\
\hline Total & 106 & \multicolumn{7}{c}{-} & & 38 & & $\mathbf{1 4 4}$ \\
\hline
\end{tabular}

Fuente: Elaboración propia

\subsection{Instrumento}

El instrumento diseñado para el trabajo de campo fue construído bajo la fusión de criterios expuestos anteriormente en los referentes teóricos (Hernández \& Fransi, 2014; Codina, et al., 2014; RodríguezMartínez, Codina, \& Pedraza-Jiménez, 2010; González-López, Bañegil \& Buenadicha, 2013), se presenta un instrumento de medida con 49 variables divididas en 10 dimensiones según la teoría y un segmento de caracterización con información particular de los cibermedios.

Tabla 2. Dimensiones del instrumento de medición en primera instancia

\begin{tabular}{|c|c|c|c|}
\hline DIMENSIÓN & VARIABLE & DIMENSIÓN & VARIABLE \\
\hline \multirow{4}{*}{$\begin{array}{l}\text { CARACTERÍSTICAS } \\
\text { DE ORIGEN }\end{array}$} & Idioma & \multirow{4}{*}{$\begin{array}{l}\text { VISIBILIDAD Y } \\
\text { POPULARIDAD }\end{array}$} & Ranking en Google \\
\hline & País & & $\begin{array}{|lll|}\begin{array}{l}\text { Trafic } \\
\text { (TRI) }\end{array} & \text { Rank } & \text { Internationa } \\
\end{array}$ \\
\hline & Ciudad & & Trafic Rank National (TRN) \\
\hline & Lenguaje de programación & & $\begin{array}{|lll|}\begin{array}{l}\text { Número } \\
\text { indizadas }\end{array} & \text { de } & \text { páginas } \\
\end{array}$ \\
\hline \multirow{3}{*}{$\begin{array}{l}\text { CARACTERÍSTICAS } \\
\text { INTERNAS } \\
\text { CALIDAD }\end{array}$} & Dominio propio & \multirow{3}{*}{$\begin{array}{l}\text { DISPONIBILIDAD } \\
\text { DEL SISTEMA }\end{array}$} & Agilidad de la web \\
\hline & Multilenguaje & & Sistema de búsqueda \\
\hline & Navegación Adaptativa & & \begin{tabular}{|lll}
$\begin{array}{l}\text { Sistema } \\
\text { avanzada }\end{array}$ & de búsqueda \\
\end{tabular} \\
\hline
\end{tabular}


RLCS, Revista Latina de Comunicación Social, 78, 457-476

[Investigación] DOI: 10.4185/RLCS-2020-1485| ISSN 1138-5820| Año 2020

\begin{tabular}{|c|c|c|c|}
\hline & Registro de usuario & & Mapa del sitio \\
\hline & Material Didáctico & \multirow{4}{*}{$\begin{array}{l}\text { EFICIENCIA } \\
\text { EFICIENCIA }\end{array}$} & Usabilidad \\
\hline \multirow{6}{*}{$\begin{array}{l}\text { CARACTERÍSTICAS } \\
\text { COMPLEMEN- } \\
\text { TARIAS }\end{array}$} & Chat virtual & & Indización \\
\hline & Suscripción a Boletines & & Experiencias del usuario \\
\hline & Tienda virtual & & Social media \\
\hline & Pasarelas de pago & \multirow[t]{4}{*}{$\begin{array}{l}\text { ACESIBILIDAD } \\
\text { TAW }\end{array}$} & $\begin{array}{l}\text { Problemas y Advertencias } \\
\text { tipo A }\end{array}$ \\
\hline & Valoración de usuarios & & $\begin{array}{l}\text { Problemas y Advertencias } \\
\text { tipo AA }\end{array}$ \\
\hline & Noticias (News) Boletín Informativo & & $\begin{array}{l}\text { Problemas y Advertencias } \\
\text { tipo AAA }\end{array}$ \\
\hline \multirow[t]{5}{*}{ SOCIAL MEDIA } & Preguntas frecuentes (FAQ) & & Suma total de Problemas \\
\hline & Facebook & \multirow[t]{7}{*}{ ENGAGEMENT } & Engagement \\
\hline & Twitter & & $\begin{array}{l}\text { Tiempo promedio en le WS } \\
\text { del usuario }\end{array}$ \\
\hline & Instagram & & Porcentaje de rebote \\
\hline & Otra red social & & Tráfico orgánico/paga \\
\hline \multirow{3}{*}{$\begin{array}{ll}\text { FIABILIDAD } & Y \\
\text { PRIVACIDAD } & \end{array}$} & Información Corporativa & & Sitios totales que enlazan \\
\hline & Información, Servicio y Condiciones de uso & & Ranking mundial \\
\hline & Protección de datos personales & & Ranking nacional \\
\hline
\end{tabular}

Fuente: Elaboración propia

27 variables de estas dimensiones fueron valoradas de forma bimodal (Freixa, Soler-Adillon, Sora \& Ribas, 2014) con presencia y ausencia de la variable de cada dimensión de cada sitio web. Y 33 variables numéricas se analizaron bajo Análisis de Componentes Principales y Análisis Factorial Exploratorio. Estos procesos se especifican en el aparatado de análisis estadístico en las siguientes líneas.

\subsection{Procedimiento}

El proceso inició con la definición del criterio de búsqueda para determinar cuántos sitios web existen en Latinoamérica que brindan el servicio de terapia psicológica online; para este objetivo se determinó la ecuación de búsqueda que permitiera arrojar resultados efectivos en los motores de búsqueda implementados. El motor de búsqueda seleccionado para realizar el rastreo fue Google Chrome con ventana modo incógnito para no sesgar la búsqueda, dado que es el motor de búsqueda con mayor cuota del mercado a nivel mundial con el 82\% en febrero del 2020 y en abril del mismo año, con mayor furor en tiempos de pandemia, Google Chrome sigue en primer lugar con 65.5\%, siendo el buscador más usado en el mundo (NetMarketShare, 2020).

Además, se definieron los términos de la ecuación de búsqueda por los cuales se haría el rastreo de los sitios web de interés para este trabajo por Google Chrome en búsqueda avanzada, separados por el operador booleano OR (Psicología OR Online OR Servicio OR Terapia OR Consulta OR Consultorio), búsqueda restringida por la región al país que se hacía referencia, en este caso cada país perteneciente a Latinoamérica. Finalmente, el criterio de búsqueda se completó con los resultados arrojados en las 10 primeras páginas de resultados de cada país. Figura 1. 


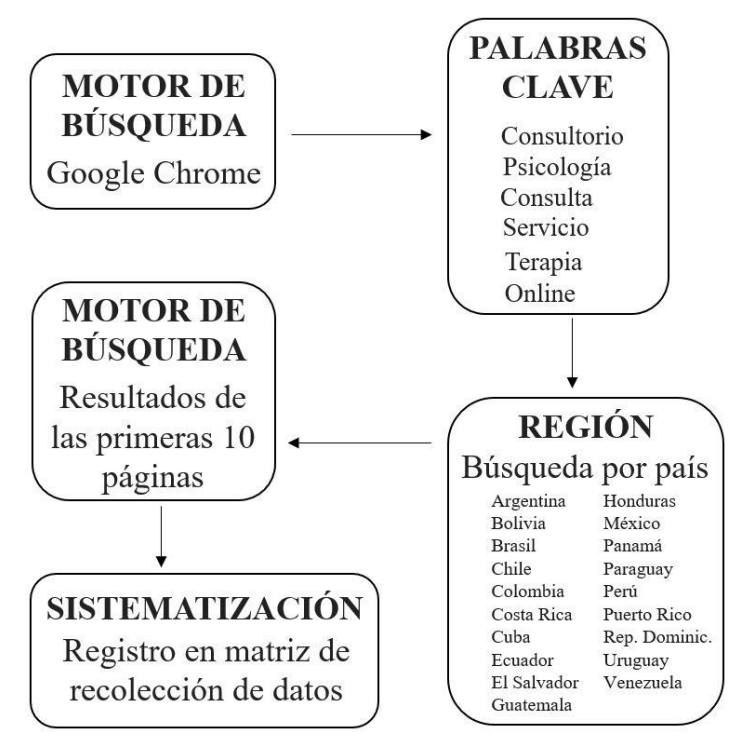

Figura 1. Equación de búsqueda

El trabajo de campo fue implementado desde el 01 de febrero hasta el 26 de marzo del 2020 y se registraron 149 sitios web en 19 de 20 países que pertenecen a Latinoamérica, teniendo en cuenta que Haití no cuenta con ningún sitio web que oferta servicios online de psicología, este fue descartado en el trabajo de campo, así mismo, se eliminaron 5 sitios web que no pertenecían al objeto de estudio, dado que se identificó que tales sitios eran plataformas de perfiles de psicólogos. Posteriormente se inició el registro y sistematización de la información de cada sitio web encontrada en la matriz de información diseñada en Excel con calificación bimodal ( 0 ó 1 ausencia o presencia) en cada variable soslayando los sesgos subjetivos de los investigadores; y otras valoraciones de corte cuantitativo según ciertas variables definidos en dicha matriz como los son el posicionamiento en Ranking (PageRank), Flujo de confianza (Trust Flow) entre otros (Ver Tabla 3); sus valoraciones son meramente cuantitativas en algunas discretas y otras continuas.

\subsection{Análisis estadístico}

Como se mencionó anteriormente, el objetivo del presente trabajo pretende construir un indicador que mida los sitios web que brindan servicios de psicología online en latinoamérica, entre otros subindices que midan la información extraída de las variables objetivas fuertemente correlacionadas en cada uno de los sitios web encontrados y debidamente sistematizados.

Cada URL sistematizada fue analizada por varios sitios web (Tabla 3) o herramientas TIC validadas científicamente en otros estudios (González-López et al., 2013; Rodríguez-Martínez, et.al., 2010; De Andrés, Lorca \& Martínez, 2010; Miranda \& Bañegil, 2004; Islam y Tsuji, 2011; Won et al., 2010; Takahashi \& Kitagawa, 2009, Tanaka et al., 2010; Yamamoto \& Tanaka, 2011) para analizar las diferentes variables propuestas en esta investigación.

Tabla 3. Recursos TIC para la evaluación de cada sitio web

\begin{tabular}{|c|c|c|}
\hline Recurso & Descripción & URL \\
\hline $\begin{array}{l}\text { W3Techs - } \\
\text { World } \\
\text { Wide Web }\end{array}$ & $\begin{array}{l}\text { Analiza el uso de tecnologías en el desarrollo del sitio web, } \\
\text { el uso de los lenguajes de programación de cada sitio; Así } \\
\text { mismo, analiza los servidores donde está alojado los sitios }\end{array}$ & https://w3techs.com / \\
\hline
\end{tabular}


RLCS, Revista Latina de Comunicación Social, 78, 457-476

[Investigación] DOI: 10.4185/RLCS-2020-1485| ISSN 1138-5820| Año 2020

\begin{tabular}{|c|c|c|}
\hline $\begin{array}{l}\text { Technology } \\
\text { Surveys }\end{array}$ & $\begin{array}{l}\text { web, el servidor web, proveedor de hosting, proveedor de } \\
\text { servicios de mail, el idioma, entre otros. }\end{array}$ & \\
\hline Google & $\begin{array}{l}\text { Motor de búsqueda de Google en modo incógnito, } \\
\text { colocando en el buscador cada URL precedida de la palabra } \\
\text { "site: o link:" de esta forma se obtienen el número de } \\
\text { resultados en segundos. }\end{array}$ & $\begin{array}{l}\text { https://www.google.com/ } \\
\text { ejemplos: } \\
\text { site:http://www.funlam.edu.co/ } \\
\text { link:https://www.funlam.edu.co/ }\end{array}$ \\
\hline Pingdom & $\begin{array}{l}\text { Tiempo / velocidad de carga, tamaño total de archivos, } \\
\text { número de respuestas, entre otros. }\end{array}$ & https://tools.pingdom.com/ \\
\hline Taw & $\begin{array}{l}\text { Test de Accesibilidad Web del centro tecnológico de la } \\
\text { información y la comunicación (CTIC), Allí se recoge } \\
\text { información sobre los errores de un sitio web discriminados } \\
\text { en número de Advertencias y número de Problemas bajo } \\
\text { tres niveles de adecuación: A; doble A (AA) y triple A } \\
\text { (AAA). }\end{array}$ & www.tawdis.net/ \\
\hline $\begin{array}{l}\text { Alexa } \\
\text { Ranking }\end{array}$ & $\begin{array}{l}\text { Popularidad de un sitio web en ranking mundial y nacional, } \\
\text { ofrece como puntuación óptima } 1 \text {. Alexa ofrece otras } \\
\text { métricas como engagemente, keywords, tasa de rebote, } \\
\text { entre otros. }\end{array}$ & https://www.alexa.com/ \\
\hline $\begin{array}{l}\text { Google } \\
\text { PageRank }\end{array}$ & $\begin{array}{l}\text { Clasificación de los sitios web en términos de popularidad a } \\
\text { través de una puntuación de } 0 \text { a } 10 \text { donde } 10 \text { es la mejor } \\
\text { puntuación; Allí mismo se obtienen métricas de Indización, } \\
\text { Autoridad del dominio, Flujo de confianza, Flujo de } \\
\text { menciones etc. }\end{array}$ & https://checkpagerank.net/ \\
\hline
\end{tabular}

Fuente: Elaboración propia

Teniendo en cuenta que el instrumento contiene 27 variables dicotómicas y 33 variables numéricas, se analizaron estos dos segmentos por separados y se procedió a realizar diferentes procedimientos; el segmento de variables dicotómicas se sometió a estudios de fiabilidad interna (George \& Mallery, 2003; Hernandez-Sampieri, Fernández \& Baptista, 2014) mediante la prueba estadística Alpha de Crombach $(\alpha)$, el coeficiente de fiabilidad compuesta (CR) en el que se consideran óptimos los valores mayores de .70 según Gefen y Straub (2005). Se calcularon las correspondientes medidas de tendencia central $(M)$ y de dispersión $(D T)$.

Además, con el segmento de variables numéricas, con el propósito de la consecusión de estos objetivos se procede a implementar el análisis multivariado como técnica principal el análisis factorial exploratorio bajo la técnica de componentes principales, teniendo en cuenta su capacidad de resumir un alto volumen de información, permitiendo identificar las diferentes dimensiones o constructos mayormente significativos para el estudio y así evitar consideraciones subjetivas.

Inicialmente se verifica la adecuación de la muestra de la matriz de correlaciones, verificando la posible factorización con la técnica de multivariante y normalización con prueba de KMO según el criterio de Kaiser-Mayer-Olkin (idoneidad de los datos) y una extracción de componentes (Guisande, Vaamonde \& Barreiro, 2011). Acto seguido, se procede a eliminar aquellas variables con extracciones bajas siguiendo a Hair et al. (1999) y Pérez (2004); estas extracciones expresan la proporción de varianza de una variable explicada por los factores seleccionados. En ese sentido, mejora el KMO y se aplica de nuevo la técnica de Análisis Factorial a las variables restantes.

\section{Resultados}

Para realizar un análisis juicioso y teniendo en cuenta la construcción del instrumento usado para la sistematización de la información de los sitios web analizados, los primeros resultados arrojados corresponden a las 26 variables de corte retracóricas (dicotómicas), las cuales fueron sometidas a 
RLCS, Revista Latina de Comunicación Social, 78, 457-476

[Investigación] DOI: 10.4185/RLCS-2020-1485| ISSN 1138-5820| Año 2020

estadísticos descriptivos y con medidas de tendencia central, que permiten analizar las frecuencias de cada variable y sus diferentes distribuciones $(D T)$, promedios $(M)$, asimetrías $(A)$ y curtosis $(K)$.

Tabla 4. Estadísticos descriptivos - variables retracóricas (dicotómicas) ( $n=144)$

\begin{tabular}{lcccc}
\hline \multicolumn{1}{c}{ Ítem } & $\boldsymbol{M}$ & $\boldsymbol{D T}$ & $\boldsymbol{A}$ & $\boldsymbol{K}$ \\
\hline 3_Dominio Propio & .95 & .216 & -4.242 & 16.222 \\
4_Multilenguaje & .11 & .315 & 2.501 & 4.315 \\
5_Navegación Adaptativa & .72 & .449 & -1.003 & -1.009 \\
6_Registro de Usuario & .31 & .465 & .818 & -1.351 \\
7_Material Didáctico & .22 & .417 & 1.35 & -.179 \\
8_Cookies & .19 & .397 & 1.56 & .441 \\
9_Chat Virtual & .13 & .332 & 2.292 & 3.298 \\
10_Suscripción a Boletines Informativos & .15 & .361 & 1.951 & 1.83 \\
11_Tienda Virtual & .35 & .48 & .616 & -1.643 \\
12_Pasarelas de Pago & .38 & .488 & .491 & -1.784 \\
13_Valoración en Estrellas & .13 & .332 & 2.292 & 3.298 \\
14_Noticias & .28 & .449 & 1.003 & -1.009 \\
15_FAQ & .31 & .462 & .853 & -1.29 \\
17_Facebook & .71 & .456 & -.926 & -1.158 \\
18_Twitter & .4 & .492 & .401 & -1.866 \\
19_Instagram & .41 & .493 & .371 & -1.889 \\
20_YouTube & .26 & .442 & 1.083 & -.84 \\
21_Otra Red Social & .52 & .501 & -.084 & -2.021 \\
23_Información Corporativa & .89 & .315 & -2.501 & 4.315 \\
24_Información de Servicio y Condiciones de Uso & .36 & .482 & .584 & -1.682 \\
25_Protección de Datos Personales & .35 & .478 & .649 & -1.602 \\
26_Experiencia de los Clientes & .27 & .446 & 1.042 \\
27_Sistema de Búsqueda & .46 & .5 & .169 & -.927 \\
28_Sistema de Búsqueda Avanzada & .07 & .255 & 3.423 \\
29_Mapa del Sitio Web & .31 & .462 & .853 & -1.999 \\
\hline
\end{tabular}

Fuente: Elaboración propia

En la tabla 4 se encuentran los resultados descriptivos, y con el propósito de reducir al máximo el error y aumentar la validez del instrumento y su confiabilidad se eliminó la variable 16 (El sitio web tiene redes sociales) dado que, por razones que su correlación corregida es de coeficiente negativo; por consiguiente, la correlación entre los ítems mejora sustancialmente y arroja un nivel de fiabilidad muy bueno con un alfa de crombah de $(\alpha=.701)$ con 25 de los 26 ítems, categorizando el instrumento como óptimo y fiable según el criterio de George y Mallery (2003); HernandezSampieri, Fernández \& Baptista, 2014; Gefen y Straub (2005).

Según los resultados, el valor de la media total es de $(\mathrm{Mt}=0.3634)$ en un rango de 0 a 1 , obteniendo el valor más bajo en $(\mathrm{I} 28=.07)$ y el más alto $(\mathrm{I} 3=.95)$; esto indica que, en un alto porcentaje los sitios web no cuentan con la mayoría de los ítems analizados, degradando la calidad de los mismos, y la variable con mayor frecuencia de presencia es el dominio propio (I3). En términos de varianzas se presentan bajas desviaciones, teniendo en cuenta que su rango oscila entre DT $=.216$ y DT $=501$ en los ítems I3 y I21 respectivamente, indicando variabilidad de los datos muy baja. Por otra parte, se encontró información atípica evidenciando que la mayoría de los datos son de carga positiva, mínimo y máximo, correspondiente a los ítems I3 $=-4.242$ y I28 $=3.423$. 
Tabla 5. Matriz de componentes rotados - Rotación varimax -Varianza total explicada. $N=144$

\begin{tabular}{|c|c|c|c|c|c|c|c|c|}
\hline \multirow[b]{2}{*}{ Factores } & \multicolumn{8}{|c|}{ Componentes } \\
\hline & Reactivos & GPrFT & $\mathbf{P A}$ & $\mathbf{A A}$ & BGPr & EA & SeTc & Extracción \\
\hline & 39VPP & 0,943 & & & & & & 0,939 \\
\hline & 43VPP & 0,935 & & & & & & 0,917 \\
\hline Google & 38VPP & 0,935 & & & & & & 0,917 \\
\hline PageRank y & 40VPP & 0,917 & & & & & & 0,897 \\
\hline Fuentes de & $42 \mathrm{C}$ & 0,88 & & & & & & 0,817 \\
\hline \multirow{3}{*}{ Tráfico } & $41 \mathrm{C}$ & 0,849 & & & & & & 0,764 \\
\hline & $44 \mathrm{C}$ & 0,849 & & & & & & 0,766 \\
\hline & $55 \mathrm{Ef}$ & 0,459 & & & & & & 0,568 \\
\hline \multirow{4}{*}{$\begin{array}{l}\text { Número de } \\
\text { Problemas A- } \\
\text { AA-AAA }\end{array}$} & $36 \mathrm{~A}$ & & 0,984 & & & & & 0,992 \\
\hline & $32 \mathrm{~A}$ & & 0,983 & & & & & 0,989 \\
\hline & $30 \mathrm{~A}$ & & 0,982 & & & & & 0,988 \\
\hline & $34 \mathrm{~A}$ & & 0,969 & & & & & 0,966 \\
\hline \multirow{4}{*}{$\begin{array}{l}\text { Número de } \\
\text { Advertencias } \\
\text { A-AA-AAA }\end{array}$} & $37 \mathrm{~A}$ & & & 0,988 & & & & 0,998 \\
\hline & $35 \mathrm{~A}$ & & & 0,973 & & & & 0,953 \\
\hline & $31 \mathrm{~A}$ & & & 0,96 & & & & 0,942 \\
\hline & $33 \mathrm{~A}$ & & & 0,959 & & & & 0,975 \\
\hline \multirow{3}{*}{ Baclinks GPr } & $47 \mathrm{I}$ & & & & 0,963 & & & 0,963 \\
\hline & $46 \mathrm{I}$ & & & & 0,962 & & & 0,966 \\
\hline & $45 \mathrm{I}$ & & & & 0,862 & & & 0,881 \\
\hline \multirow{3}{*}{$\begin{array}{c}\text { Engagement } \\
\text { de Alexa }\end{array}$} & $53 \mathrm{Eg}$ & & & & & 0,753 & & 0,61 \\
\hline & $52 \mathrm{Eg}$ & & & & & 0,578 & & 0,562 \\
\hline & $54 \mathrm{Eg}$ & & & & & 0,55 & & 0,648 \\
\hline \multirow{9}{*}{$\begin{array}{c}\text { Sitios enlazan } \\
\text { y Tiempo de } \\
\text { Carga }\end{array}$} & $49 \mathrm{I}$ & & & & & & 0,79 & 0,735 \\
\hline & & & & & & & & \\
\hline & $57 \mathrm{Ef}$ & & & & & & $-0,593$ & 0,569 \\
\hline & $\begin{array}{l}\text { Autovalor } \\
\%\end{array}$ & 28,071 & 16,619 & 16,061 & 12,207 & 6,729 & 4,979 & 84,666 \\
\hline & Varianza & 1,16963 & 0,69246 & 0,66921 & 0,50863 & 0,28038 & 0,20746 & \\
\hline & \multicolumn{6}{|c|}{ Kaiser-Meyer-Olkin de adecuación de muestreo } & & 0,702 \\
\hline & & & & \multirow{3}{*}{\multicolumn{3}{|c|}{ Test de Esfericidad de Bartlett }} & $X^{2}$ & 6941,6 \\
\hline & & & & & & & gl & 276 \\
\hline & & & & & & & Sig. & 0,000 \\
\hline
\end{tabular}

Fuente: Elaboración propia

No obstante, al momento de someter las 32 variables numéricas al estudio de Análisis Factorial Exploratorio (AFE), se detectaron extracciones de los ítems con coeficientes muy bajos por debajo de (<.5) (Ferrando \& Anguiano-Carrasco 2010; González et al., 2013; Lloret-Segura et al., 2014) estos ítems fueron I22, I45, I48, I49, I50, I51, I58, I59, I60 y I61, los cuales fueron eliminados para dar mejor resultado en la varianza extractada; estas extracciones expresan la proporción de varianza de una variable explicada por los factores extraídos. En este sentido, los resultados en KMO = .702 (superior a .6) y la prueba de esfericidad de Bartlett $=.000$ (menor que .05) (Tabla 5) por lo que la hipótesis nula se constrasta, por consiguiente el modelo factorial es adecuado para explicar el fenómeno.

Al aplicar la técnica multivariante de componentes principales (Guisande, Vaamonde \& Barreiro, 2011) con extracción Varimax para determinar el nivel de correlación entre las variables, resultaron 6 componentes con una varianza extractada del $84.67 \%$, el componente que mejor explica el fenómeno 
es el componente 1 denominado Google PageRank y Fuentes de Tráfico (GPrFT) con el 28.07\% representando la mayoría de la información (Tabla 5).

\subsection{Indicador de Medición en Psicología Online - IMPOL}

El indicador IMPOL es un indicador general construído a partir de las variables seleccionadas en el AFE anterior; y se expresa mediante la siguiente fórmula matemática:

$$
\mathrm{IMPOL}=\sum_{\iota=0}^{n} \mathcal{S} \mathcal{J}_{\iota} \quad x \%{ }^{\text {Apriori }}
$$

1. El factor 1 está compuesto por resultados obtenidos de Google PageRank y Fuentes de tráfico en mayor medida, este factor se denominó GPrFT.

2. El factor 2 está compuesto por los resultados obtenidos por las variables que hacen referencia a los problemas bajo los tres niveles de adecuación (A, AA y AAA), este factor se denominó Problemas de Adecuación - PA.

3. El factor 3 está compuesto por los resultados obtenidos por las variables que hacen referencia a las advertencias bajo los tres niveles de adecuación (A, AA y AAA), este factor se denominó Advertencias de Adecuacuación - AA.

4. El factor 4 está compuesto por los resultados obtenidos por las variables que hacen referencia a los Backlinks de Google PageRank, este factor se denominó BGPr.

5. El factor 5 está compuesto por los resultados obtenidos por las variables que hacen referencia a los indicadores de Engagement de Alexa, este factor se denominó EA.

6. El factor 6 está compuesto por los resultados obtenidos por las variables que hacen referencia a los indicadores de Sitios que enlazan y el Tiempo de carga, este factor se denominó SeTc.

Tabla 6. Top 10 de los sitios web en función al indicador de Psicología Online IMPOL. $n=144$

\begin{tabular}{ccll}
\hline$\#$ & IMPOL & & NOMBRE DEL SITIO WEB \\
\hline 1 & 0,4629 & Integra Médica & Chile \\
2 & 0,4918 & Dr.consulta & Brasil \\
3 & 0,5670 & Consejo Regional de Psicología CRP 3 & Brasil \\
4 & 0,5860 & Zen Klub & Brasil \\
5 & 0,5919 & Psicología viva & Brasil \\
6 & 0,6000 & AMAPSI & México \\
7 & 0,6166 & FEPRA Federación de psicólogos de la Republica de Argentina & Argentina \\
8 & 0,6214 & AMADAG & Chile \\
9 & 0,6238 & Psicología Online CEPSI Honduras & Honduras \\
10 & 0,6551 & Mundo Psicólogos & Argentina \\
11 & 0,6740 & IEPP & Colombia \\
\hline
\end{tabular}

Fuente: Elaboración propia

Al momento de aplicar el indicador IMPOL a cada una de las URL objeto de estudio de este trabajo, se estandarizan los resultados obtenidos por cada una, con una ponderación que va desde 0 a 1 , siendo 0 el mejor resultado, y el resultado entre más alejado del 0 indica peor resultado. En este orden de ideas, en la tabla 6 se identifican las 10 primeras URL (sitios web) con mejores puntuaciones frente al indicador IMPOL. Como se puede observar se hallaron 2 sitios web que ofrecen psicología online de nacionalidad chilena, 4 brasileras, 1 mejicana, 2 argentinas y 1 hondureña (Tabla 6). 
RLCS, Revista Latina de Comunicación Social, 78, 457-476

[Investigación] DOI: 10.4185/RLCS-2020-1485| ISSN 1138-5820| Año 2020

Teniendo en cuenta la revisión de la literatura, se concluye que los componentes se relacionan por sus contenidos conceptuales en estos factores, y de esta forma se reconfiguran las dimensiones en solo tres factores o sub-indicadores de la manera que se describe a continuación:

El factor 1 GPrFT y el factor $4 \mathrm{BGPr}$, estos factores hacen referencia al posicionamiento web, suponiendo entre ambos una varianza explicada del fenómeno del $40.27 \%$. De esta forma se reconstruye un nuevo sub-indicador denominado "Indicador de Posicionamiento Web - IPW"

- Indicador IPW $=.28071(\mathrm{GPrFT})+.12207(\mathrm{BGPr})$ con un nivel de representatividad de importancia del $47.6 \%$

Tabla 7. Top 10 de los sitios web en función del Indicador de Posicionamiento web IPW.

\begin{tabular}{|c|c|c|c|}
\hline \# & IPW & NOMBRE DEL SITIO WEB & PAÍS \\
\hline 1 & 0,1381 & Dr. Consulta & Brasil \\
\hline 2 & 0,1460 & Integra Médica & Chile \\
\hline 3 & 0,1481 & $\mathrm{CES} / \mathrm{JF}$ & Brasil \\
\hline 4 & 0,1583 & Consejo Regional de Psicología CRP 3 & Brasil \\
\hline 5 & 0,1681 & FEPRA Federación de psicólogos de la República de Argentina & Argentina \\
\hline 6 & 0,1741 & AMAPSI & MEX- \\
\hline 7 & 0,1767 & Zen Klub & Brasil \\
\hline 8 & 0,1936 & Psicología viva & Brasil \\
\hline 9 & 0,1959 & Psicólogos & COL- \\
\hline 10 & 0,1969 & Mundo Psicólogos & Argentina \\
\hline 11 & 0,1976 & AMADAG & Chile \\
\hline
\end{tabular}

Fuente: Elaboración propia

El factor 2 PA y el factor 3 AA, hacen referencia a la accesibilidad, suponiendo entre ambos una varianza explicada del fenómeno en un $32.68 \%$. De esta forma se reconstruye un nuevo subindicador denominado "indicador de Acesibilidad IA"

- Indicador IA = .16619(PA) +.16061(AA) con un nivel de representatividad de importancia del $38.6 \%$

Los valores obtenidos en cada sitio web se interpretan al contrario que los otros sub-indicadores, el valor entre más alejado del 0 tiene mayor accesibilidad, dado por su condición de los indicadores de problemas y advertencias al arrojar por cada URL cifras muy elevadas en términos generales.

Tabla 8. Top 10 de los sitios web en función del Indicador de Acesibilidad - IA.

\begin{tabular}{ccll}
\hline$\#$ & IA & & \multicolumn{1}{c}{ NOMBRE DEL SITIO WEB } \\
\hline 1 & 1,0213 & InAltum Clínica de Superación Personal \\
2 & 1,2569 & Terapia virtual-Psicólogos en línea & Argentina \\
3 & 1,2569 & Oriéntate & Rolombia \\
4 & 0,6808 & Psicólogos la Guía & Argentina Dom \\
5 & 0,4300 & Juan Roque & Argentina \\
6 & 0,2005 & La casa Ámbar & Panamá \\
7 & 0,1816 & Psicología On-IIn CEPSI & Honduras \\
8 & 0,1757 & Tu psicóloga & Colombia \\
9 & 0,1667 & Adriano Gosuen & Brasil \\
10 & 0,0973 & Terapia web & Argentina \\
\hline
\end{tabular}

Fuente: Elaboración propia 
RLCS, Revista Latina de Comunicación Social, 78, 457-476

[Investigación] DOI: 10.4185/RLCS-2020-1485| ISSN 1138-5820| Año 2020

El factor 5 EA y el factor 6 SeTc, hacen referencia al Engagement y a la Eficiencia, suponiendo entre ambos una varianza explicada del fenómeno del $11.71 \%$. De esta forma se reconstruye un nuevo subindicador denominado "Indicador de Eficiencia para el Engagement IEE"

- Indicador IEE $=.0673(\mathrm{EA})+.0498(\mathrm{SeTc})$ con un nivel de representatividad de importancia del $13.8 \%$

Tabla 9. Top 10 de los sitios web en función del Indicador de Eficiencia para el Engagement IEE.

\begin{tabular}{ccll}
\hline$\#$ & IEE & & NOMBRE DEL SITIO WEB \\
\hline 1 & 0,0211 & CFP & PAÍS \\
2 & 0,0492 & Psicología On-línea CEPSI & Honduras \\
3 & 0,0568 & Integra Médica & Chile \\
4 & 0,0679 & AMADAG & Chile \\
5 & 0,0750 & Psicología estratégica & Colombia \\
6 & 0,0756 & Chile psicólogos & Chile \\
7 & 0,0773 & Psicología viva & Brasil \\
8 & 0,0874 & R\&A Psicológos & MEX- \\
9 & 0,0902 & Dr. Consulta & Brasil \\
10 & 0,1256 & Zen Klub & Brasil \\
\hline
\end{tabular}

Fuente: Elaboración propia

De todos los países latinoamericanos analizados que cuentan con sitios web para brindar psicología online, se clasificó de forma tipo ranking con los resultados obtenidos, dado que se estudiaron todos los 144 sitios web existentes con esta condición evitando la soslayación de sesgos. En la Tabla 10 se aprecian los resultados globales con la aplicación de todos los indicadores luego de determinar los promedios por cada país, se hace referencia al indicador global IMPOL donde se identificaron los países mejor valorados.

Tabla 10. Posición que ocupa la nacionalidad frente a los indicadores propuestos.

\begin{tabular}{lcccc}
\hline \multicolumn{1}{c}{ PAÍS } & IMPOL & IPW & IA & IEE \\
\hline Bolivia & 0,0079 & 0,2780 & 0,0185 & 0,0002 \\
Brasil & 0,3749 & 0,1143 & 0,0230 & 0,0463 \\
Honduras & 0,4722 & 0,1471 & 0,0373 & 0,0482 \\
Ecuador & 0,6832 & 0,2190 & 0,0290 & 0,1221 \\
Chile & 0,7931 & 0,2549 & 0,0210 & 0,1922 \\
Argentina & 0,8165 & 0,2489 & 0,0219 & 0,4553 \\
México & 1,0799 & 0,3688 & 0,0136 & 0,4921 \\
Colombia & 1,1239 & 0,3961 & 0,0234 & 0,4266 \\
Rep. Dominicana & 1,6868 & 0,5742 & 0,0382 & 1,1714 \\
Perú & 2,0375 & 1,0993 & 0,0307 & 1,6783 \\
Puerto Rico & 2,0860 & 1,1624 & 0,0248 & 1,7129 \\
Panamá & 2,2340 & 1,5142 & 0,0135 & 0,7765 \\
Venezuela & 2,4579 & 1,0960 & 0,0476 & 1,0624 \\
Paraguay & 2,6796 & 2,5655 & 0,0204 & 1,6726 \\
El Salvador & 2,7222 & 1,2951 & 0,0481 & 1,8673 \\
Uruguay & 2,7905 & 1,5315 & 0,0487 & 0,6774 \\
Costa Rica & 3,3720 & 2,3016 & 0,0577 & 1,2045 \\
Guatemala & 3,6351 & 2,6283 & 0,0470 & 2,8855 \\
\hline
\end{tabular}

Fuente: Elaboración propia

Finalmente se presenta en la Tabla 11 los 6 países con mejores resultados obtenidos luego de la aplicación de cada indicador a cada URL y de cada país, hay que tener presente que no todos los 
países tiene la misma cantidad de URL analizadas y por esta razón se encuentra en la Tabla 10 a Bolivia en el primer lugar, pues este país cuenta con solo 5 sitios web en contraste de países como Brasil, Argentina y Colombia que cuentan con 22, 26 y 28 sitios web respectivamente, que brindan servicios de psicología online en tiempos de pandemia.

Tabla 11. Posición que ocupa la nacionalidad según los 144 sitios web en función de los indicadores propuestos.

\begin{tabular}{lcccccc}
\hline \multicolumn{1}{c}{ PAÍ́ } & IMPOL & IPW & IA & IEE & Prom & Escalafón \\
\hline Argentina & 2 & 2 & 1 & 5 & 2,50 & 2 \\
Brasil & 1 & 1 & 5 & 1 & 2,00 & 1 \\
Chile & 3 & 3 & - & 2 & 2,67 & 3 \\
Colombia & 5 & 4 & 2 & 3 & 3,50 & 4 \\
Honduras & 4 & - & 3 & 4 & 3,67 & 5 \\
México & 6 & 5 & - & 6 & 5,67 & 6 \\
Panamá & - & - & 4 & - & 4,00 & - \\
\hline
\end{tabular}

Fuente: Elaboración propia

\section{Discusión}

Por medio de los indicadores propuestos en el presente trabajo puntualmente el Indicador de Medición de Psicología Online (IMPOL), el Indicador de Posicionamiento Web (IPW), el Indicador de Accesibilidad (IA) y el Indicador de Eficiencia para el Engagement (IEE), se han identificado los principales sitios web en América Latina que brindan los servicios en Psicología online. De tal modo que los sitios web de nacionalidad brasilera son los que tienen mejores resultados en los países de América Latina.

El presente trabajo evidencia similitudes con estudios propiamente mencionados en el estado del arte de la investigación, se logró determinar los conceptos relacionados con los estudios realizados por Hernández y Fransi, (2014); Codina, et al., (2014); Rodríguez-Martínez, Codina, y Pedraza-Jiménez, (2010); González-López, Bañegil y Buenadicha, (2013), de los cuales se sustrajo las variables que mejor se ajustaban al modelo propuesto y para el sector de la psicología online, teniendo en cuenta la conveniencia del estudio y los resultados arrojados por el método estadístico implementado. Por otro lado, se analizaron variables de valoración cualitativas y cuantitativas propuestas por los estudios de estos mismos estudios, obteniendo óptimos resultados que satisfacen los objetivos propuestos.

Los resultados confirman que, aunque en los Indicadores implementados y sin conservar proporcionalidad entre la cantidad de sitios web que brindan el mismo servicio, son los países que menos tienen sitios web en psicología online en tiempos de pandemia los que arrojan resultados desfavorables; de esta forma se evidencia que entre mayor oferta del servicio en psicología online, mayor será el esfuerzo por los psicólogos que brindan este tipo de servicios virtuales, quienes deben ofrecer mayor eficiencia, accesibilidad y posicionamiento web a sus públicos potenciales, teniendo en cuenta la alta competencia en el mercado en el campo de la psicología online.

A pesar de las condiciones pandémicas que atraviesa el mundo actualmente, y la latente necesidad de las personas para acceder a terapias psicológicas online o por teleconferencia, los sitios web carecen de varios aspectos de calidad web si se aplica el índice que propone González et al., (2013) y Rodríguez-Martínez et al., (2010); este fenómeno se presenta por el gran aumento de plataformas 
web que con sus desarrollos y estructuras web preestablecidas no dinamizan la diversificación del sector en aspectos de calidad web, seguridad, accesibilidad y posicionamiento web, sobre todo en este último aspecto que se evidenciaron resultados muy bajos, pues de los 144 sitios web analizados solo el 4,86\% logró pasar la barrera de la mitad (5) del Ranking en conteo de 0 a 10.

\section{Conclusiones}

Se concluye, que la oferta de servicios en psicología online no es la más fiable y carece de recursos que favorecen la compra de estos servicios de manera confiable y segura, hay que tener presente que para la cantidad de países analizados no se encuentra una oferta satisfactoria de estos servicios en términos de cantidad, exceptuando a Colombia, Brasil, Argentina y Chile, que son los que más oferta tienen de este tipo de servicios online, en especial en la época pandémica que es cuando se requieren mayor cobertura en la prestación de este servicio virtual; aunque estudios recientes confirman que el uso de videoconferencias ha sido bajo en épocas de pre-pandemia COVID-19, este tipo de servicios ha venido creciendo sustancialmente durante la crisis (Helou et al., 2020)

Es importante la generación de nuevos sitios web en psicología online, pero más importante es la generación de estrategias de posicionamiento web que los profesionales puedan implementar tácticamente para ser competitivos en el mercado virtual, y aquellos sitios web existentes en la actualidad deben fortalecer su accesibilidad, seguridad, popularidad, visibilización y posicionamiento web para generar confianza en sus públicos objetivos. Por esta razón es importante que se requiera de inversión en el desarrollo web experimentado para optimizar los recursos de software y realizar un adecuado desarrollo particularizado, es decir, con características únicas que generen valor y de esta forma potenciar la competitividad.

El presente estudio valida estadísticamente los indicadores aquí presentados, se analizaron todos los sitios web que brindan el servicio de este estudio, se implementó metodológicamente análisis factorial que permitió diseñar indicadores exclusivos para este sector en específico, se validaron las variables dicotómicas arrojando un alfa satisfactorio para determinar que el instrumento es fiable y consisitente. Del mismo modo, se sugiere para futuras investigaciones, se considere analizar desde el ámbito de contenido otro instrumento que complemente el presente estudio, y de esta forma ampliar el rango de análisis para implementar un modelo análítico de sitios web para la categoría de Psicología online. Se sugiere implementar otros motores de búsqueda para no soslayar los hallazgos en futuros estudios.

Finalmente se propone que se implementen los indicadores propuestos a otros sectores específicamente en Latinoamérica para validar aún más los indicadores, y sea esta la oportunidad de estudiar las percepciones de los usuarios y robustecer los indicadores por medio de ecuaciones estructurales SEM. Hay que tener presente, que los indicadores propuestos son un piloto de evaluación de las plataformas o servicios de psicología online en función de protección a los usuarios y posibles fraudes.

\section{Bibliografía}

Arana, J. M., Meilán, J. J. G., y Pérez, E. (2006). El concepto de psicología. Entre la diversidad conceptual y la conveniencia de unificación. Apreciaciones desde la epistemología. 8(1), 33. https://www.redalyc.org/comocitar.oa?id=80280107 
Baena, A., Quesada, M. y Vogt, F. (2008). La e-psicología: posibilidades y limitaciones de las tecnologías de la información y de la comunicación en la terapia a distancia. Revista de Psicoterapia, 19(73), 119-140.

Barnes, S. J., y Vidgen, R. (2002). An evaluation of cyber-bookshops: The WebQual method. International Journal of Electronic Commerce, 6(1), 11-30. https://doi.org/10.1080/10864415.2001.11044225

Bauer, C., y Scharl, A. (2000). Quantitative evaluation of Web site content and structure. Internet Research: Electronic Networking Applications and Policy, 10(1), 31-43. https://doi.org/10.1108/10662240010312138

BID. (2010). Ciencia, Tecnología e Innovación en América Latina y el Caribe. Un compendio estadístico de indicadores. New York, USA: BID.

Bouzas-Lorenzo, R., y Mahou L. X. M. (2013). El estudio de la funcionalidad de los portales gubernamentales: Una propuesta metodológica desde la e-salud. Gestión y política pública, 22(spe), 259-305.

Brown, F. W. (1998). Rural telepsychiatry. Psychiatric Services, 49, 963-964. https://doi.org/10.1176/ps.49.7.963

Castells, M. (2009). Comunicación y poder. Madrid. Alianza Editorial.

Codina, L., Pedraza, R., Díaz, N. J., Rodríguez-Martínez, R., Pérez-Montoro, M., y Cavaller-Reyes, V., (2014). Sistema Articulado de Análisis de Cibermedios (SAAC): Una propuesta sobre el qué y el cómo para estudiar medios de comunicación digitales. Hipertext.net. 12, DOI: https://doi.org/10.2436/20.8050.01.13

Conesa F. M., Aguinaga O. E. y Hernández M. J. (2011). An evaluation of the quality of health web pages using a validated questionnaire. Aten Primaria. 43(1) 33-40. https://doi.org/10.1016/j.aprim.2010.01.007

Cristóbal-Fransi, E. y Marimon-Viadiu, F. (2011). "La gestión del supermercado virtual: tipificación del comportamiento del cliente online". Investigaciones Europeas de Dirección y Economía de la Empresa. 17(1) 93-112. https://doi.org/10.1016/S1135-2523(12)60046-X

Charria O. V., Sarsosa P. K., y Arenas O. F. (2011). Construcción Y Validación De Contenido De Un Diccionario De Las Competencias Genéricas Del Psicólogo: Académicas, Profesionales Y Laborales. Interdisciplinaria: Revista de Psicología y Ciencias Afines, 28(2), 299-322.

De Andrés, J., Lorca, P., y Martínez, A. B. (2010). Factors influencing web accessibility of big listed firms: an international study. Online Information Review, 34(1), 75-97. https://doi.org/10.1108/14684521011024137

Domínguez F. D. (2007). Sobre la intención de la etnografía virtual. Teoría de la Educación. Educación y Cultura en la Sociedad de la Información, 8(1),42-63

Echeburúa, E., y de Corral, P. (2010). Adicción a las nuevas tecnologías y a las redes sociales en jóvenes: un nuevo reto. Adicciones, 22(2), 91-95. https://doi.org/10.20882/adicciones.196 
Espinar, E., Zych, I., y Rodríguez-Hidalgo, A. J. (2015). Ciberconducta y dependencia emocional en parejas jóvenes. Psychology, Society, \& Education, 7(1), 41-55. https://doi.org/10.25115/psye.v7i1.539

Ferrando, P. J. y Anguiano-Carrasco, C. (2010). El análisis factorial como técnica de investigación en psicología. Papeles del Psicólogo, 31(1), 18-33.

Fornell, C., y Larcker, D. F. (1981). Evaluating structural equation models with unobservable variables and measurement error. Journal of Marketing Research, 18(1), 39-50. https://doi.org/10.2307/3151312

Freixa, P., Soler-Adillon J., Sora C., y Ribas J. I., (2014). Aportaciones Del découpage Interactivo En La Lectura Y análisis De Audiovisuales Interactivos De Los Cibermedios. Hipertext.Net 12, doi:274410. Recuperado de: https://bit.ly/35gXKe7

Gefen, D., y Straub, D. (2005). A practical guide to factorial validity using PLSgraph: Tutorial and annotated example. Communications of the AIS, 16(1), 91-109. https://doi.org/10.17705/1CAIS.01605

George, D. y Mallery, P. (2003). SPSS/PC step by step: a simple guide and reference. Wadsworth Publishing Co. Belmont, CA. EEUU.

González L. O. Bañegil, P. T. y Buenadicha, M. M. (2013) El índice de calidad web como instrumento objetivo de medición de la calidad de sitios web corporativos. Revista Investigaciones Europeas de Dirección y Economía de la Empresa. 19(1) 16-30. https://doi.org/10.1016/j.iedee.2012.07.004

Guisande, C., Vaamonde, A., \& Barreiro, A. (2011). Tratamiento de Datos con R, Statistica y SPSS. España. Ediciones Díaz de Santos. P. 667 - 757.

Hair, J. F., Anderson, R. E., Tatham, R. L., \& Black, W. C. (1999). Análisis multivariante. Madrid: Prentice Hall.

Helou, S., Helou, E., Abou-Khalil, V., Wakim, J., Helou, J., Daher, A., Hachem, C. (2020). The Effect of the COVID-19 Pandemic on Physicians' Use and Perception of Telehealth: The Case of Lebanon. J. Environ. Res. Public Health, 17(13), 4866. https://doi.org/10.3390/ijerph17134866

Hernández-Sampieri, R., Fernández, C. \& Baptista, P. (2014). Metodología de la Investigación, México, D.F.: Editorial McGraw Hill.

Hernández S. F., y Fransi, C. E. (2014). Estado de la cuestión y nuevas perspectivas en la evaluación de la calidad percibida en los cibermedios. Historia $Y$ Comunicación Social, 18, 15-27. https://doi.org/10.5209/rev_HICS.2013.v18.44222

Islam, A., y Tsuji, K. (2011). Evaluation of usage of university websites in Bangladesh. Journal of Library \& Information Technology, 31(6), 469-479. 
Loiacono, E. T., Watson, R. T. y Goodhue, D. L. (2007). WebQual: an instrument for consumer evaluation of web sites. International Journal of Electronic Commerce, 11(3), 51-87. https://doi.org/10.2753/JEC1086-4415110302

Liu, S., Yang, L., Zhang, C., Xiang, Y.-T., Liu, Z., Hu, S., Zhang, B. (2020). Online mental health services in China during the COVID-19 outbreak. The Lancet Psychiatry 7(4) 17-18. https://doi.org/10.1016/S2215-0366(20)30077-8

Lloret-Segura, S., Ferreres-Traver, A., Hernández-Baeza, A. y Tomás-Marco, I. (2014) El análisis factorial exploratorio de los ítems: una guía práctica, Anales de psicología, 30(3) 1151-1169 http://dx.doi.org/10.6018/analesps.30.3.199361

Miranda, F. J., y Bañegil, T. M. (2004). Quantitative evaluation of commercial web sites: An empirical study of Spanish firms. International Journal of Information Management, 24(4), 313 328. https://doi.org/10.1016/j.ijinfomgt.2004.04.009

Morato, J., Sánchez-Cuadrado, S., y Fernández-Bajón, M.-T. (2016). Tendencias en El Perfil Tecnológico Del Profesional De La Información. El Profesional de La Información, 25(2), 169178. https://doi.org/10.3145/epi.2016.mar.03

Netmarketshare (2020). Browser Market Share. United States. Recuperado de: https://bit.ly/2XETKDu

Noone, C., McSharry, J., Smalle, M., Burns, A., Dwan, K., Devane, D., Morrissey, E.C. (2020). Video calls for reducing social isolation and loneliness in older people: A rapid review. Cochrane Database of Systematic Reviews 5, 1-40.

Organización Panamericana de la Salud (2016). La eSalud en la Región de las Américas: derribando las barreras a la implementación. Resultados de la Tercera Encuesta Global de eSalud de la Organización Mundial de la Salud. Washington, D.C. OPS, 2016

Ortega Ruíz, R., Del Rey, R. y Sánchez, V. (2012). Nuevas dimensiones de la convivencia escolar juvenil: ciberconducta y relaciones en la red: ciberconvivencia. Ministerio de Educación, Cultura y Deporte. Universidad de Córdoba: España Secretaría General Técnica.

Palacios, M., y Díaz, N. (2009). Online Journalism: Research Methods. A multidisciplinary approach in comparative perspective. Bilbao. Servicio Editorial de la Universidad del País Vasco.

Parasuraman, A., Zeithaml, V. A., y Malhotra, A. (2005). E-S-QUAL: a multi-item scale for assessing electronic service quality. Journal of Service Research, 7(3), 213-233. https://doi.org/10.1177/1094670504271156

Pérez, L. C. (2004) Técnicas de análisis multivariante de datos. Editorial Pearson.

Petnji, L., Marimon, F. y Casadesús, M. (2012). "Assessing e-service quality: the current state of ES-QUAL”, Total Quality Management Business Excellence, 23, 1363-1378

Rappa M. (2005). Business models on the web. Recuperado de: https://bit.ly/2REcn6u [consultado 12 abril 2020]. 
Rodríguez-Martínez, R., Codina, L., y Pedraza-Jiménez, R. (2010). Cibermedios y web 2.0: modelo de análisis y resultados de aplicación. El profesional de la información. 19, (1), 35-44. https://doi.org/10.3145/epi.2010.ene.05

Sanmartín, M. P., Ávila, H. K., Vilora, N. C. y Jabba, M. D. (2016). Internet de las cosas y la salud centrada en el hogar. Revista Científica Salud Uninorte 32(2) 1-13

Soto, J. S., y Gómez, C. G. (2018). Avances en realidad virtual e intervenciones en psicología clínica. Tesis Psicológica, 13(1), 1-15. Recuperado de https://bit.ly/3esv9ra

Wolfinbarger, M., y Gilly, M. C. (2003). eTailQ: Dimensionalizing, measuring and predicting etail quality. Journal of Retailing, 79(3), 183-198. https://doi.org/10.1016/S0022-4359(03)00034-4

Won, K., Jeong, O., y Lee, S. (2010). On social websites. Information Systems, 32(2), 215-236. https://doi.org/10.1016/j.is.2009.08.003

Takahashi, T., y Kitagawa, H. (2009). A ranking method for web search using social bookmarks. Lecture Notes in Computing Science, 5463, 585-589.

Tanaka, K., Nakamura, S., Ohshima, H., Yamamoto, Y., Yanbe, Y., y Kato, M. (2010). Improving search and information credibility analysis from interaction between web1.0 and web2.0 content. Journal of Software, 5(2), 154-159.

Valdés P. L. (2013). Evaluación de sitios web de salud: método y aplicación. No Solo Usabilidad, $12,1-18$

Vázquez Casielles, R.; Río-Lanza, A.B.; Suárez-Álvarez, L. (2009). "Las agencias de viaje virtuales: ¿Cómo analizar la calidad de e-servicio y sus efectos sobre la satisfacción del cliente?" Universia Business Review, 24, 122-143

Voces-Merayo, R. (2007). “Por qué la Web debe ser accesible?”. Hipertext.net, 2007, n. 5. https://bit.ly/34AgPss

Yamamoto, Y., y Tanaka, K. (2011). Enhancing credibility judgment of web search results. CHI '11 Proceedings of the 2011 annual conference on Human factors in computing systems, 1235-1244.

Yang, H.; Zhang, X. (2019). Investigating the Effect of Paid and Free Feedback About Physicians' Telemedicine Services on Patients' and Physicians' Behaviors: Panel Data Analysis. J. Med. Internet Res. 21, e12156. https://doi.org/10.2196/12156

Yoo, B., y Donthu, N. (2001). Developing a scale to measure perceived quality of an Internet shopping site (SITEQUAL). Quarterly Journal of Electronic Commerce, 2(1), 31-46.

Zeithaml, V. A. (2000). Service quality, profitability and the economic worth of customers: What we know and what we need to learn. Journal of the Academy of Marketing Science, 28(1), 67-85. https://doi.org/10.1177/0092070300281007

Zeithaml, V. A. (2002). Service excellence in electronic channels. Managing Service Quality, 12(3), 135-138. https://doi.org/10.1108/09604520210429187 
RLCS, Revista Latina de Comunicación Social, 78, 457-476

[Investigación] DOI: 10.4185/RLCS-2020-1485| ISSN 1138-5820| Año 2020

Zeithaml, V. A., Parasuraman, A., y Malhotra, A. (2002). Service quality delivery through web sites: A critical review of extant knowledge. Journal of the Academy of Marketing Science, 30(4), 362375. https://doi.org/10.1177/009207002236911

Zhou, X., Snoswell, C., Harding, L., Bambling, M., Edirippulige, S., Bai, X, Smith, A. (2020). The Role of Telehealth in Reducing the Mental Health Burden from COVID-19. Telemed J E Health. 26(4) 377-379. https://doi.org/10.1089/tmj.2020.0068

\section{AUTORES:}

\section{Carlos Andrés Trejos-Gil}

Facultad de Comunicación Publicidad y Diseño, programa de Comunicación Social. Universidad Católica Luis Amigó. Magíster Internacional en Dirección Estratégica; Magister en Administración y Dirección de Empresas (MBA). Especialista en Alta Gerencia. Publicista. Ingeniero Administrativo. Docente Investigador en comunicación social, administración de empresas, mercadeo, publicidad, TIC y Learning. Consultor empresarial en áreas administrativas, comerciales, financieras y de mercadeo. Capacitador y formador en TIC y tecnologías digitales. Áreas de investigación: TIC; Marketing Digital; B-Learning; Ciberculturas; Administración; Marketing; Ciencias sociales; Tecnología.

carlos.trejosgi@amigo.edu.co

Orcid ID: https://orcid.org/0000-0002-6769-3396

Google Scholar: https://scholar.google.com/citations?user=46Gr7u4AAAAJ\&hl=es\&oi=ao

ResearchGate: https://www.researchgate.net/profile/Carlos_Trejos_Gil

Academia.edu: https://funlam.academia.edu/CARLOSANDRESTREJOSGIL

\section{Huber Yesid Castro Escobar}

Docente en el área de TIC y Educación, Docente Investigador en Educación, TIC y Medios Audiovisuales y Líder del grupo de Investigación Calidad de Vida, de la Universidad Católica Luis Amigó. Magister en Comunicación de la Universidad del Norte. Licenciado en Informática y Medios Audiovisuales de la Universidad de Córdoba. Áreas de investigación: TIC, Educación y Comunicación para el Cambio Social.

huber.castroes@amigo.edu.co

Orcid ID: https://orcid.org/0000-0002-8714-2487

Google Scholar: https://scholar.google.com/citations?user=1OUtSkIAAAAJ\&hl=es

\section{Omar Augusto Amador Sánchez}

Profesional en Psicología, especialista en Terapia Cognitiva y Magister en Psicología de la Universidad de San Buenaventura-Medellín. Con mas de 10 años de experiencia en atención clínica individual, docente e investigador en Psicopatología, psicología clínica cognitiva y capacitador en temas afines. https://orcid.org/0000-0001-6013-8480 Áreas de investigación, Psicología Clínica, Suicidio, psicología online, psicopatología, ciencias sociales y del comportamiento. Investigador del grupo de investigación Neurociencias Básicas y Aplicadas (NBA) de la Universidad Católica Luis Amigó. omar.amadorsa@amigo.edu.co

Orcid ID: https://orcid.org/0000-0001-6013-8480

Google Scholar: https://scholar.google.com/citations?user=2EdSYP0AAAAJ\&hl=es\&oi=sra

ResearchGate: https://www.researchgate.net/profile/Omar_Amador5 